\section{SOBRE LA NOMENCLATURA TAXONÓMICA Y SISTEMÁTICA DE LOS APICOMPLEJOS}

\author{
ABOUT THE TAXONOMIC AND SYSTEMATIC \\ NOMENCLATURE OF APICOMPLEJOS
}

\section{Dalmiro Cazorla-Perfetti ${ }^{1, a}$}

Sr. Editor. Hemos leído con especial interés el artículo de Silva-Díaz et al. (1) en donde hacen un estudio acerca de la frecuencia de "coccidios intestinales" en 325 niños atendidos en un hospital de Perú, y la comparación de dos métodos de diagnóstico para Cryptosporidium spp. En líneas generales coincidimos en los tópicos abordados por dichos investigadores en su artículo; sin embargo, aprovechamos esta oportunidad para hacer comentarios y ampliar los conocimientos acerca de la posición y nomenclatura taxonómica y sistemática de los apicomplejos que infectan al humano, en particular de los denominados usualmente como "coccidios intestinales" (y por extensión a los que infectan otros tipos de tejidos u órganos), y que en el citado artículo se les señalan como "protozoarios".

Tradicionalmente, los "coccidios intestinales" incluyendo Cryptosporidium spp. (agente causal de criptosporidiosis), Cystoisospora belli (= Isospora belli) (agente etiológico de cystoisosporiosis) y Cyclospora cayetanensis (agente causal de ciclosporiosis), se han considerado como pertenecientes al grupo taxonómico de los protozoarios (Reino Protista, subreino Protozoa) ${ }^{(2)}$. Sin embargo, especialmentehacialadécadade 1990 coneladvenimiento y aplicación de las técnicas de la microscopía electrónica, moleculares y las herramientas filogenéticas-cladistas y de la bioinformática, se ha revelado que los eucariotas simples (usualmente denominados como protistas) es un grupo muy heterogéneo y parafilético; de allí que en la actualidad los apicomplejos se consideran de pertenecer al reino Chromista (sensu Ruggiero et al.) (3), en el cual se incluyen las algas cromofitas así como también varios grupos taxonómicos que anteriormente se les incluyó dentro de los protozoarios y hongos (actualmente: Reinos Protozoa y Fungi) ${ }^{(3)}$; en ambos reinos destacan grupos taxonómicos de importancia médico-zoonótica como Blastocystis spp. (agente etiológico de blastocistosis) o el ciliado intestinal Balantidium coli (agente etiológico de balantidiosis), y un grupo de pseudohongos (Oomicetos) del género Pythium spp. ( $P$. insidiosum y $P$. aphanidermatum, agentes causales de pitiosis) ${ }^{(2,3,4)}$.

\footnotetext{
1 Laboratorio de Entomología, Parasitología y Medicina Tropical (LEPAMET), Centro de Investigaciones Biomédicas, Decanato de Investigaciones, Universidad Nacional Experimental "Francisco de Miranda". Santa Ana de Coro, Venezuela.

Biólogo, Doctor of Science

Recibido: 30/04/2017 Aprobado: 07/06/2017 En línea: 28/06/2017
}

Citar como: Cazorla-Perfetti D. Sobre la nomenclatura taxonómica y sistemática de los apicomplejos. Rev Peru Med Exp Salud Publica. 2017;34(2):351. doi: 10.17843/rpmesp.2017.342.2849
La aplicación de estas técnicas mencionadas ha sido tan impresionante y reveladora que hasta en el nivel taxonómico de clase, subclase y orden se ha detectado que Cryptosporidium spp. se encuentra más relacionado con las gregarinas (Gregarinomorphea, Cryptogregaria, Cryptogregarida) que con Cyclospora spp. y Cystoisospora spp. (Coccidiomorphea, Coccidea, Eimerida) ${ }^{(5)}$.

A la luz de todo lo discutido, entonces, es recomendable revisar, actualizar y homogenizar la nomenclatura taxonómicay sistemática de varias taxa de microorganismos parásitos, incluyendo los de hábitos entéricos, en las revistas biomédicas de nuestro medio; esta propuesta tiene la finalidad que se denomine y describa más apropiadamente, por ejemplo, como cromistas en vez de protozoarios a microrganismos apicomplejos como los ya señalados y discutidos, u otros como los plasmodios humanos (Plasmodium spp.) agentes causales de malaria o paludismo, o a Toxoplasma gondii, agente etiológico de la toxoplasmosis ${ }^{(2)}$. Sin embargo, se debe acotar que aún persisten muchas controversias y debates entre los investigadores del área acerca de los límites entre los Reinos que agrupan actualmente los seres vivos ${ }^{(3)}$, por lo que, en los estudios taxonómicos y sistemáticos, quiérase o no, siempre existe un nivel o grado de subjetividad.

Fuentes de financiamiento: autofinanciado

Conflictos de interés: el autor refiere no tener conflictos de interés en la publicación de este artículo.

\section{REFERENCIAS BIBLIOGRÁFICAS}

1. Silva-Díaz H, Campos-Flores H, Llagas-Linares J, LlantasCancino D. Coccidiosis intestinal en niños admitidos en un hospital de Perú y comparación de dos métodos para la detección del Cryptosporidium spp. Rev Peru Med Exp Salud Publica. 2016; 33(4):739-44. Doi:10.17843/rpmesp.2016.334.2560

2. Botero D, Restrepo M. Parasitosis humanas. 5a ed. Medellín, Colombia: Corporación para Investigaciones Biológicas; 2012.

3. Ruggiero M, Gordon D, Orrell T, Bailly N, Bourgoin T, Brusca R, et al. 2015. A higher level classification of all living organisms. PLoS ONE. 10(4): e0119248. Doi:10.1371/ journal. pone.0119248

4. Calvano T, Blatz P, Vento T, Wickes B, Sutton D, Thompson $\mathrm{E}$, et al. Pythium aphanidermatum infection following combat trauma. J Clin Microbiol. 2011;49(10):3710-13. Doi:10.1128/ JCM.01209-11

5. Cavalier-Smith T. Gregarine site-heterogeneous $18 \mathrm{~S}$ rDNA trees, revision of gregarine higher classification, and the evolutionary diversification of Sporozoa. Eur J Protistol. 2014; 50(5): 472-95. Doi:10.1016/j.ejop.2014.07.002

Correspondencia: Dalmiro Cazorla-Perfetti

Dirección: Laboratorio de Entomología, Parasitología y Medicina Tropical (LEPAMET), Centro de Investigaciones Biomédicas, Decanato de Investigaciones, Universidad Nacional Experimental "Francisco de Miranda", Apartado 7403, Coro, Venezuela.

Teléfono: 0058-268-2521668.

Correo electrónico: cdalmiro@gmail.com 